\title{
The structure and evolution of a skyway network
}

\author{
Arthur Huang a and David Levinson ${ }^{\mathrm{b}}$ \\ University of Minnesota, Minneapolis, MN 55455, USA
}

Received 30 July 2012 / Received in final form 29 November 2012 Published online 29 January 2013

\begin{abstract}
We study the structure and evolution of the downtown Minneapolis, Minnesota skyway network. Developed by private building-owners, the network evolved from tree-like to grid-like over the course of 50 years. We find that decentralized forces with the goal of maximizing individual buildings' profitability shaped the network. Our analysis shows that a building with greater office size, a sign of greater accessibility, was more likely to be connected earlier. The distribution of existing skyway segments is found to follow a power-law function of the average degree, closeness, and eigenvector centralities of the vertices. We further explain and model the evolutionary process using an agent-based model. The simulation results suggest that the model replicates the network structure and its evolutionary process.
\end{abstract}

\section{Introduction}

There has been a growing interest in modeling the evolution of transportation networks. Examples include airline networks [1,2], railways [3,4], subways [5], highways [6], street network [7,8], and other surface road infrastructure [9-11]. Models examining the mechanism of network growth can be grouped into three categories. The first is the graphic-theoretic approach where each edge is presumably born with a probability, such as the exponential model [12], preferential attachment [13,14], Markov graphs [15,16], and the Newman-Gastner model [17]. The second is the network design approach where given a set of specifications networks are built to optimize an objective, such as minimizing detour [18], maximizing the transportation potential between two locations $[19,20]$, or minimizing total transportation costs [21-23]. The third is the agent-based approach where competitive or cooperative agents build edges with local objectives. Examples include modeling the evolution of trails in urban green spaces $[24,25]$, self-organization of road networks [26], traffic jam probability [6], and the dynamics of land use [27]. Gastner [17] contends that the first approach, concentrating on topological features, often neglects the geographical and spatial aspects of the networks $[4,28,29]$. In comparison, the second and third approaches tend to consider geography, supply, demand, and built environment conditions. The difference between the second and third approach is whether there is a centralized planner

\footnotetext{
a e-mail: huang284@umn.edu

b e-mail: dlevinson@umn.edu
} 
and how the rules are made. Comprehensive reviews of measuring network structure and modeling spatial networks can be found in [30-32].

We argue that modeling real-world transportation networks should be contextsensitive, which includes assessing who are the decision-makers, what are their goals, what is the environment, and what are the constraints. In modeling transportation networks, it is important to consider the nexus between networks and land use, which happens "in particular places, at a variety of scales (international, intercity, metropolitan, sub-metropolitan), for different technologies (pedestrian ways, roads, rails, maritime, and aviation), at different points in history" [33]. Models should be further verified by sufficient fine-scale data [34]. The challenges of modeling lie in "micro-macro integration, handling spatio-temporally explicit data, capturing humanenvironment relationships, and bridging the qualitative-quantitative divide" [35]. Here incorporating the context of network growth, we analyze the spatio-temporal data of the Minneapolis skyway network and study its evolutionary mechanism. We also propose an agent-based model to replicate the network growth process.

\section{Empirical background}

The downtown Minneapolis, Minnesota skyway network is the longest skyway network in North America. Such a network enables pedestrians to move more efficiently between connected buildings while avoiding weather and street traffic [10]. Similar to ordinary roads in terms of connecting destinations, the skyway network differs from other surface transportation networks in the following aspects. First, it is above and aligned with the street level, and only connects adjacent buildings. Second, it only allows pedestrian traffic. Third, although planned through the cooperation between private businesses and the city government, the skyway segments are privately financed and owned.

The idea of building skyway segments to connect second-level corridors within buildings in downtown Minneapolis first emerged in developer Les Park's plan of a covered plaza raised 4.25 meters over Nicollet Avenue in the 1950s [10]. As in other US cities, post World War II Minneapolis witnessed the fast development of suburbs and the relative decline of the central business district. Les Park's plan was intended to combat this trend by increasing accessibility and business opportunities within downtown Minneapolis. Although this plan was not eventually implemented, the idea of building skyways was inherited by other plans. In 1962 when the NorthStar Center opened, it featured the city's first skyway segment which connected the Center with its adjacent Northwestern National Bank building. As this center became profitable, downtown business leaders who realized the opportunities that skyways could provide began to invest in building skyway segments [36]. In 1969 three more skyway segments were built. Arguably the most captivating additions to the Skyway System were the four skyway segments connecting the IDS Center to four adjacent buildings as it opened in 1973. The IDS Center later quickly became a new landmark for downtown Minneapolis [36]. From 1962 to 1977, the skyway segments were all built in the adjacent area of the NorthStar Center. After 1977, the system grew rapidly and expanded to other blocks of downtown. The network expansion slowed down after 1992 and stopped in 2002 (aside from one segment opened in late 2011). The evolution of the skyway system can be categorized into birth (1962-80), growth (1980-92), and mature (1992-now) phases [10].

The skyway data used for analysis were developed in [10] who employed the following procedure for digitizing the skyway network: first download a street map of downtown Minneapolis in GIS format and create vertices at the center of a block. Then manually add skyway segments over the street grid in ArcGIS. In digitizing the 

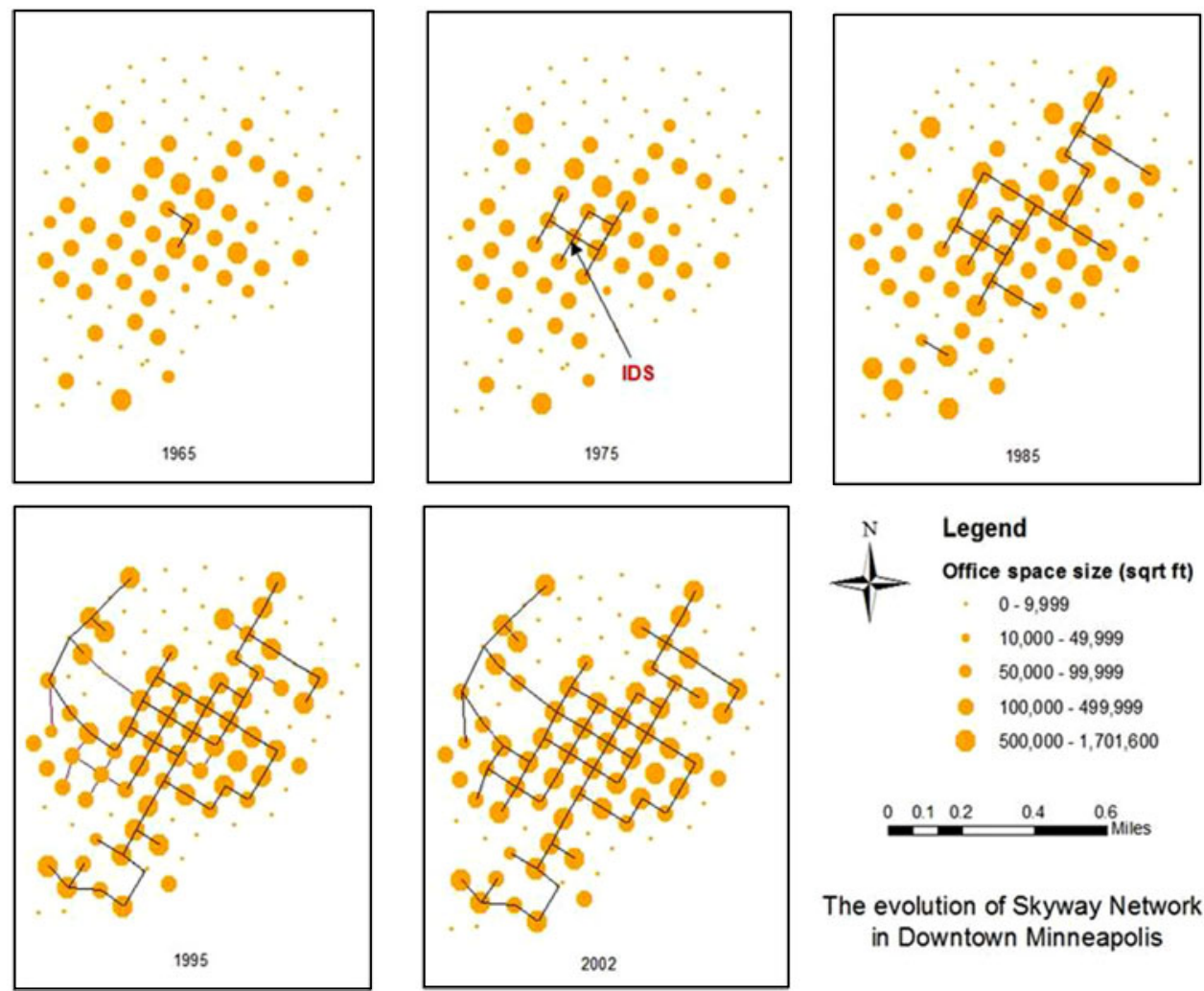

The evolution of Skyway Network in Downtown Minneapolis

Fig. 1. The evolution of the Minneapolis skyway network from 1965 to 2002. Vertices represent blocks and edges indicate skyway segments. The size of an octagon symbolizes the office space of a building or the parking space if it is a parking lot. The network first evolved around the center of downtown (the IDS Center) and expanded to buildings on the periphery, growing from tree-like structure to grid-like structure.

network, assume that the skyway segments are straight and connect the midblock of one building to another. The data on current building floor areas were downloaded from [37] and the City of Minneapolis and Hennepin County's property finder and information websites. More information about the data can be found in [10].

\section{Network structure}

Figure 1 shows the evolution of the network topology, where vertices represent blocks and edges indicate skyway segments. The size of an octagon symbolizes the office space of a building. Bigger office buildings seem more likely to be connected earlier. By observation, the network structure evolved from tree-like, to star-like, and ultimately to grid-like wherein the highest degree of connections is 4 . Figure 2 further plots the changes of the number of vertices and edges, average degree of connections, average path length, and total network length from 1962 to 2011. These parameters largely display an S-shape distribution, consistent with the phase changes described in [10]. In Fig. 2-c, there were a few jumps for the average number of connections from 1974 to 1993 because the network then consisted of two disconnected components, with one having more segments and the other having fewer than 4 edges. The network became reconnected in 1994 . 

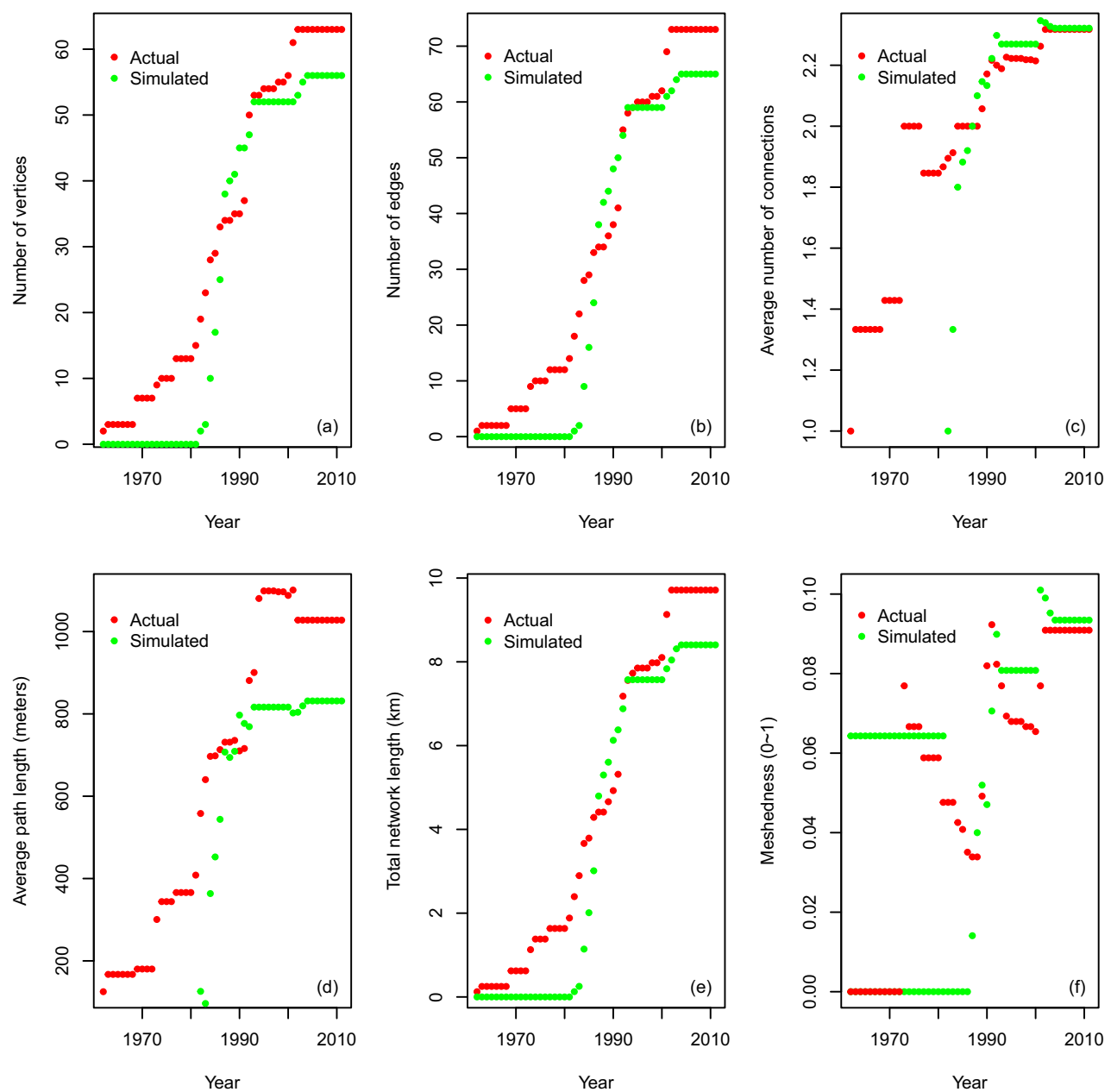

Fig. 2. The structural characteristics of the Minneapolis skyway network (1962-2011).

We use the meshedness coefficient $(M)$ to quantify how tree-like or grid-like a planar network is. It is measured as [38]:

$$
M=\frac{m-n+1}{2 n-5}
$$

where $m$ is the number of edges and $n$ is the number of vertices. $M$ varies from 0 (a complete tree) to 1 (a complete planar graph). The larger $M$ is, the more grid-like a network is. Figure 2-f reveals that the network by and large evolves from tree-like to grid-like, which comports with our observation in Fig. 1. The rise and fall of meshedness between 1974 and 1993 were because the network was disconnected. During that period, when measuring these variables we only consider the major component of the network which approximates the whole network.

We examine the average degree, closeness, betweenness, and eigenvector centralities of the vertices. When measuring the centralities, the network is constructed as an undirected and unweighted graph. Degree centrality measures the connectivity of 
a vertex. For vertex $i$, the degree centrality equals [39]:

$$
D_{i}=\frac{\tau_{i}}{n-1}
$$

where $\tau_{i}$ is the degree of vertex $i$ and $n$ is the number of vertices.

Closeness centrality measures to which degree vertex $i$ is close to all other vertices along the shortest paths [40]. The closeness centrality of vertex $i$ in graph $G$ is defined as:

$$
C_{i}=\frac{n-1}{\sum_{j \in G ; i \neq j} d_{i j}}
$$

where $d_{i j}$ is the shortest path length (measured by the number of hops) between vertex $i$ and $j$.

Betweenness centrality measures the fraction of shortest paths between pairs of vertices that pass the vertex of interest. The betweenness centrality of vertex $i$ is defined as [41]:

$$
B_{i}=\frac{1}{(n-1)(n-2)} \sum_{j, k \in G ; j \neq k \neq i} \frac{\sigma_{j k}(i)}{\sigma_{j k}}
$$

where $\sigma_{j k}$ is the number of shortest paths between $j$ and $k . \sigma_{j k}(i)$ is the number of shortest paths between $j$ and $k$ that pass $i$.

Eigenvector centrality measures the influence of a vertex by assigning eigenvector centrality scores to all vertices in the network. The methodology for calculating eigenvector centrality of a vertex is delineated in [42].

As the skyway network became more grid-like, the mean centrality indices of the vertices decreased over time. We further investigate the relationship between the probability of existing skyway segments and the centralities over the course of its evolution. In Fig. 3 we can see that the distribution of existing skyway segments follows a power-law function of degree, closeness, and eigenvector centralities, with $R^{2}>0.8$. The decay parameters respectively equal $-1.251,-1.763,-3.062$, and -2.22 . The curves indicate that the creation of new edges is correlated with the topological features of the network. More skyway segments being built in the network are associated with lower average centralities for the buildings.

We want to understand how the structure of the skyway network is related to its historical context and geographical constraints. To shed light on this, we take two steps. The first is conceptual; the second is theoretical.

\section{A conceptual framework}

A review of the history of the Minneapolis skyway network reveals that this network was built bottom up, driven by individual investors' presumably aiming to maximize profits. We sketch a conceptual framework to describe the forces that foster the network growth in Fig. 4, where individual investors are influenced by a set of incentives, disincentives, and geographic constraints regarding whether or not to build skyway segments. Such individuals' efforts gradually shape the structure of the skyway network which further induces incentives and disincentives for building more segments. The incentives include accessibility, satisfaction from employees working in the buildings, and business opportunities. The disincentives include construction cost and some investors' inertia to wait for other investors to build skyways to connect to their own buildings. The geographic constraints include planarity (in our case), aligning with the layout of streets, and buildings which are for non-commercial use and thus cannot 

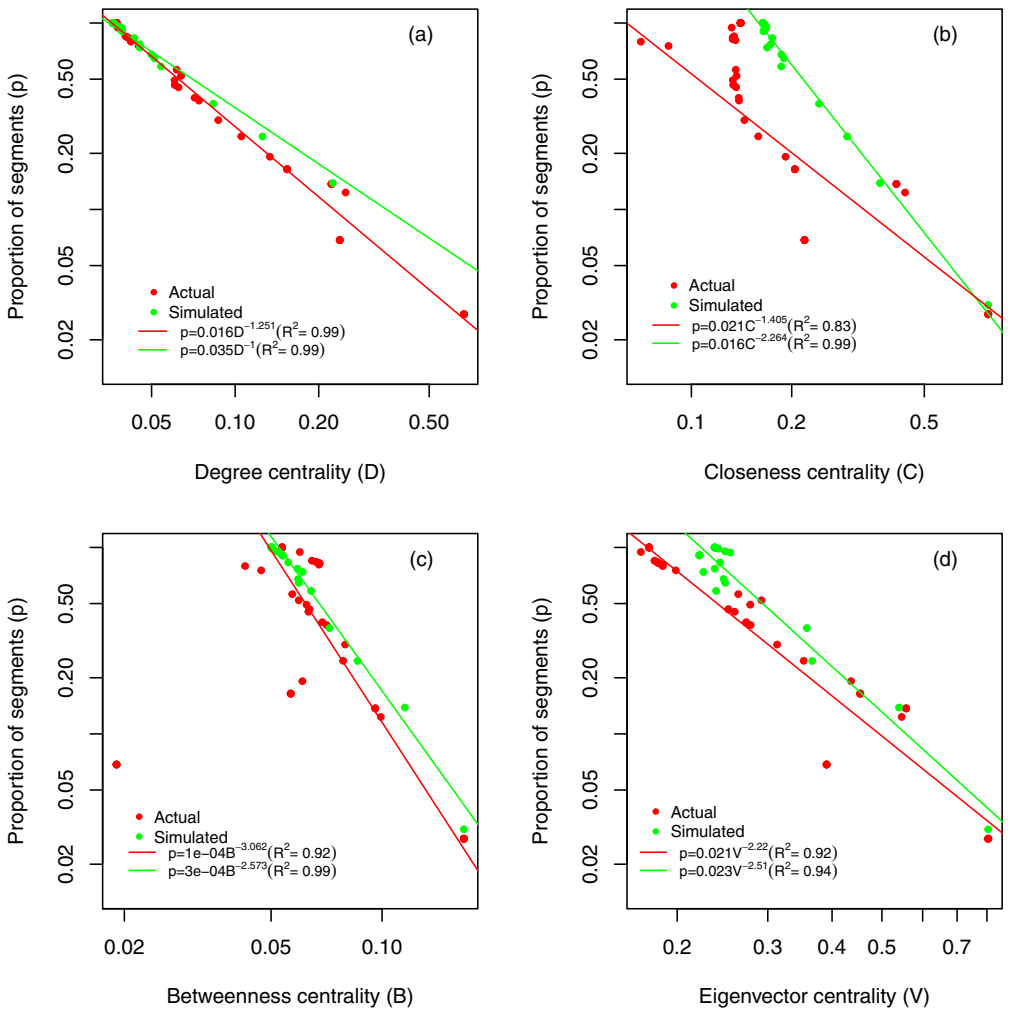

Fig. 3. The relationships between the proportion of existing skyway segments and network centrality measures. The distribution of existing skyway segments is a power-law function of average degree, closeness, and eigenvector centralities of the vertices.

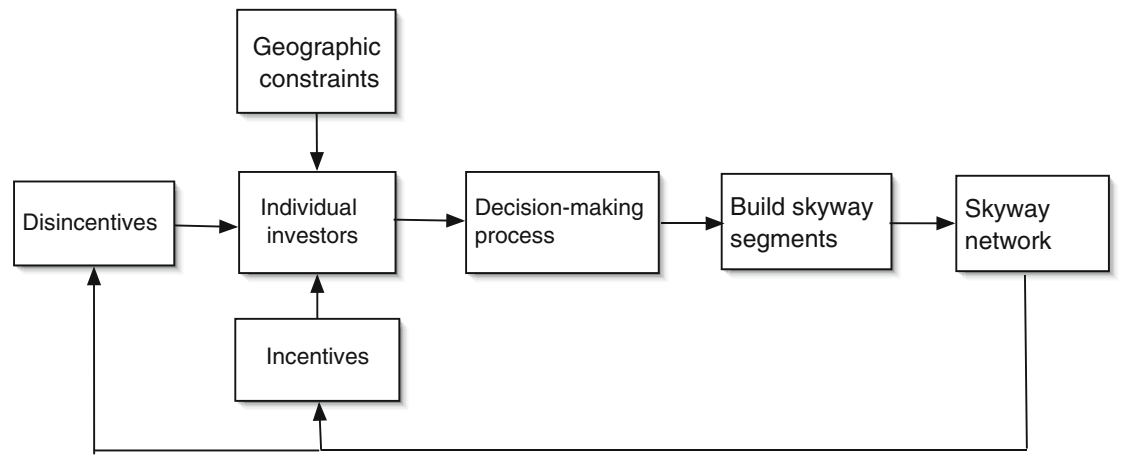

Fig. 4. A conceptual model describing the building process of the Minneapolis skyway network.

be connected. The network construction process ceases when the disincentives become greater than the incentives for all investors. Therefore, this is a system with positive and negative feedbacks.

\section{An agent-based model}

Given the incentives, disincentives, and geographic constraints, we propose an agentbased model that explains the structure and evolution of the network. The edges of 
the network are skyway segments, and the vertices are buildings. Let's assume the value of accessibility for the owner of vertex $i$ to build a new segment $l$ in iteration $t$ equals:

$$
A_{i}(l, t)=\sum_{j=1}^{J_{i}} w \phi_{j}(t) \zeta_{i j}(t)^{-\delta}
$$

where $J_{i}$ is the number of adjacent buildings around building $i$ and $\zeta_{i j}(t)$ is the shortest travel distance (actual path length measured in meters) between building $i$ and building $j$ along the network in iteration $t . \delta$ is the distance decay parameter, indicating that a longer travel path lowers the value of accessibility. $w$ is the value of accessing one employee. $\phi_{j}(t)$ represents the total number of employees in building $j$ in iteration $t$, which is assumed to be proportional to building $j$ 's floor area. Since the employees' data are not available for verification, this assumption is only approximately true, but it serves as a plausible start (see a similar study in [43]). In addition, since a building's floor area evolved over time, $\phi_{j}(t)$ in each iteration is updated using building $j$ 's floor area in a corresponding year.

For the owner of building (vertex) $i$, the incentive to build segment $l$ is measured as the increased value of accessibility due to this new segment. The disincentive is the cost of building and maintaining segment $l$ which is presumably proportional to its length. The marginal benefit for the owner of building $i$ to build segment $l$ in iteration $t$ equals the difference between the incentive and disincentive. It can be described as:

$$
\Delta b_{i}(l, t)=A_{i}(l, t)-A_{i}(l-1, t-1)-c \cdot \gamma_{l}
$$

where $\gamma_{l}$ is the length of segment $l$, and $c$ is the construction cost per unit length of a segment. Among all possible segments to be built, the owner of building $i$ selects the segment that provides the highest marginal benefit. If the highest marginal benefit is greater than zero, segment $l$ will be built; otherwise not built. This is thus a locally selfish, myopic optimization, maximizing short-term benefit for the owner of a building. In deciding the segment candidates for each building owner, we rule out the segments which are not built because of specific constraints (e.g., government administrative building or historical structures).

To quantify the accuracy of our modeled network, we measure the network's $F_{1}$ score which is the harmonic mean of the precision $\alpha$ and recall $\beta$. $\alpha$ is the number of correct segments divided by the number of all simulated segments and $\beta$ is the number of correct segments divided by the number of actual segments. $F_{1}$ equals [44]:

$$
F_{1}=2 \cdot \frac{\alpha \cdot \beta}{\alpha+\beta}
$$

$F_{1}$ ranges from 0 to 1 . The higher $F_{1}$ is, the more similar the simulated network is as the actual.

To find the simulated network that best matches the actual, we test different values of $\delta$ from 0.5 to 0.8 (with step size 0.02 ), $w$ from 1 to 2 (with step size 0.1 ), and $c$ from 180 to 350 (with step size 10) and run each set of parameters for 50 iterations. How $F_{1}$ changes with $\delta, c$, and $w$ is shown in Fig. 5. We find that the maximum $F_{1}$ score equals 0.82 which is produced by two scenarios. The first scenario is $\delta=0.68, w=1.4$, and $c=320$. The second scenario is $\delta=0.7, w=1.2$, and $c=260$. Further details about finding the two scenarios are shown in Fig. 6 .

We can use the first scenario as an example to illustrate a simulated network (given different sequences of decision-making in each iteration, the network topology can be slightly different). Figure 7 displays the generated skyway network vis-à-vis the actual skyway network in its mature stage. The actual network has 73 edges 


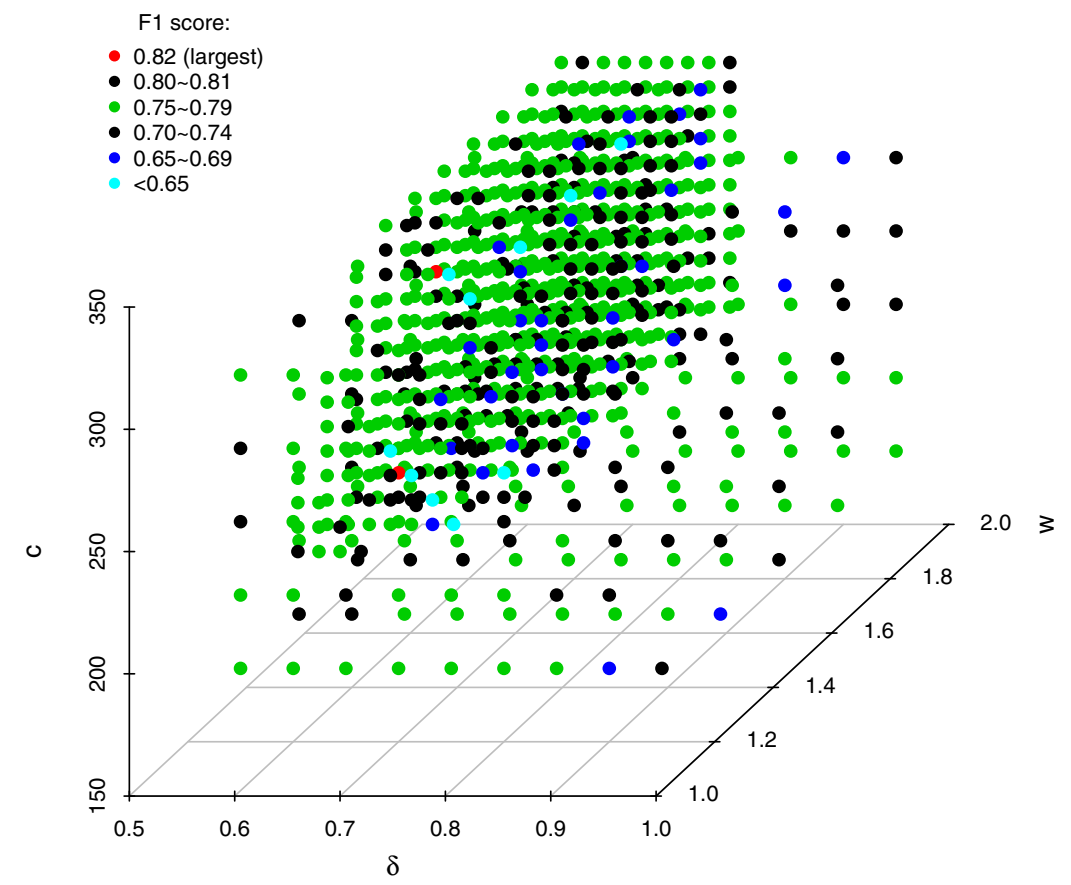

Fig. 5. The behavior of F1 score as it changes with $\delta, c$, and $w$.
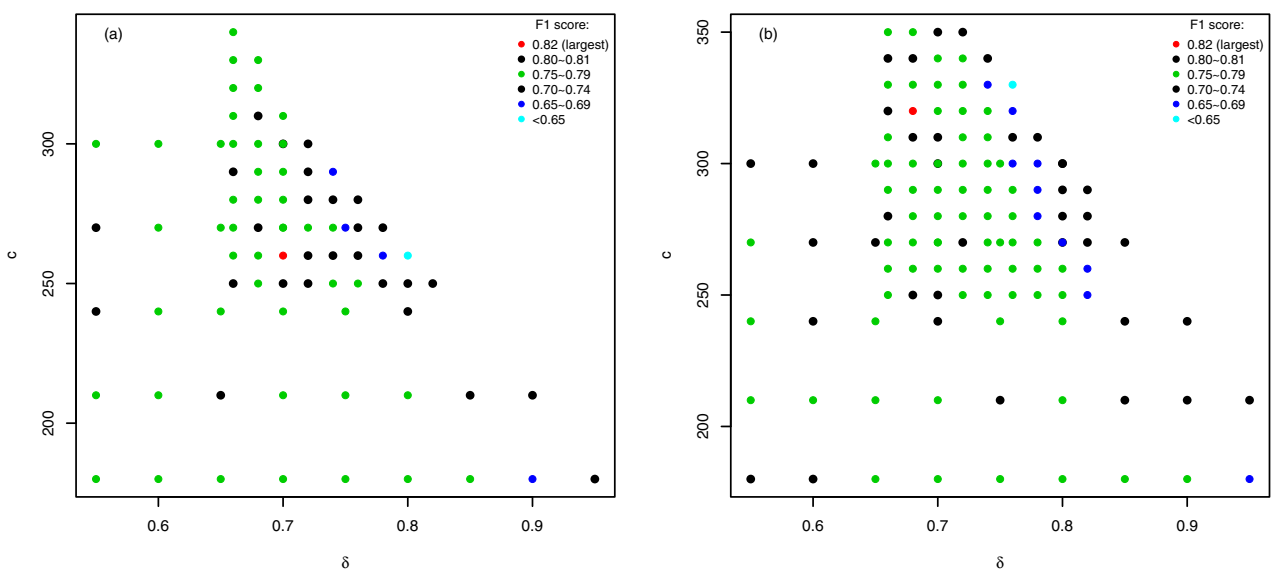

Fig. 6. (a) The distribution of $\mathrm{F} 1$ score when $w=1.2$. The largest $\mathrm{F} 1$ score is produced when $\delta=0.7, w=1.2$, and $c=260$. (b) The distribution of F1 score when $w=1.4$. The largest $\mathrm{F} 1$ score is produced when $\delta=0.68, w=1.4$, and $c=320$.

and the simulated network has 66 edges, 57 of which match the actual network. The major segments around the IDS Center are all reproduced. We further compare the structural characteristics of the simulated network with the actual network over time (Fig. 2). The evolutionary trends of vertices, edges, average number of connections, average path length, and total network length for the simulated network are similar as the actual, although the curves in the simulated network are steeper. In addition, the distribution of existing segments in the simulation network also behaves as a 


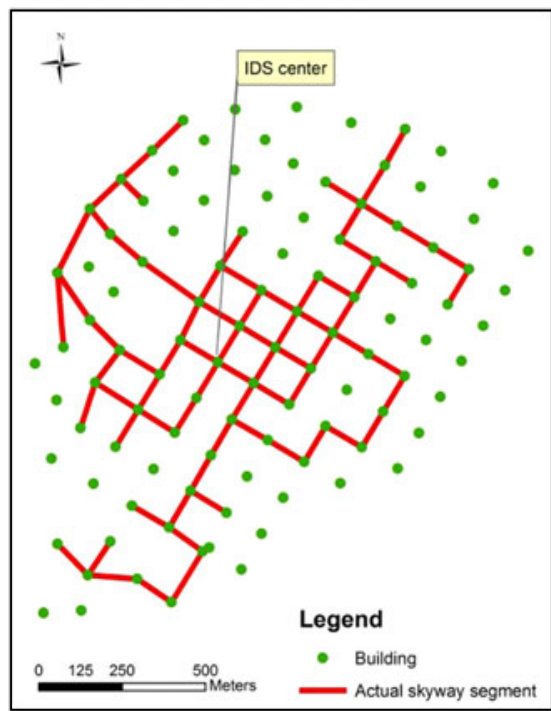

The actual skyway network

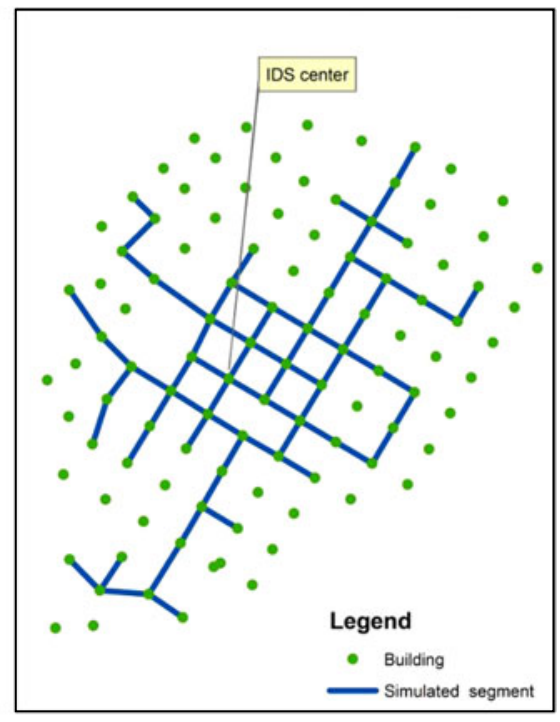

The simulated skyway network

Fig. 7. A comparison of the actual Minneapolis skyway network with a simulated network with the best goodness of fit $(\delta=0.68, w=1.4$, and $c=320)$.

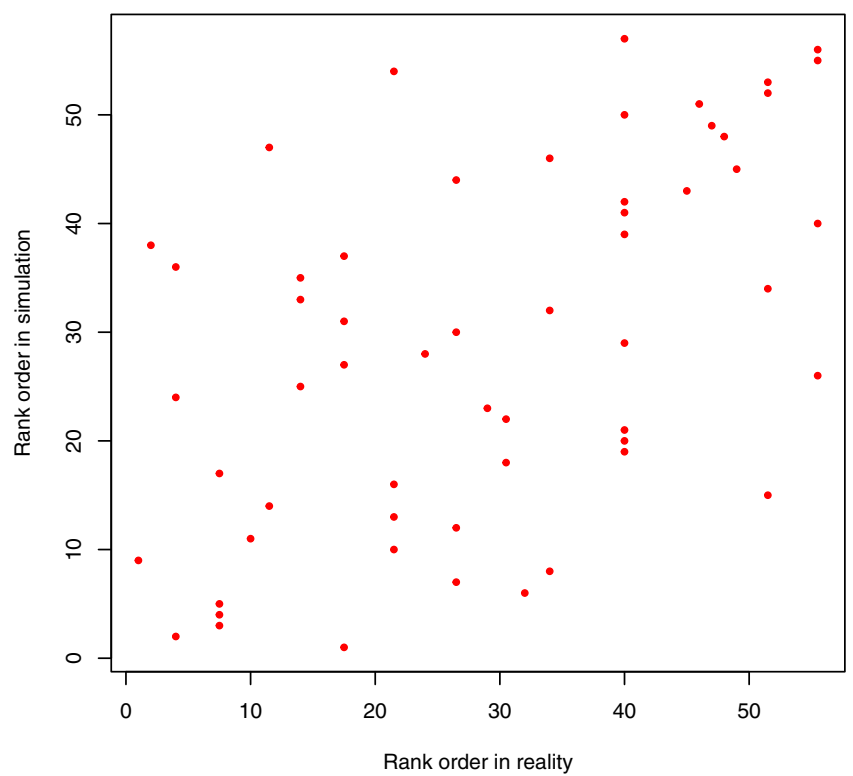

Fig. 8. Rank order in reality versus rank order in simulation. The Spearman coefficient equals 0.53 .

power-law function of centralities (Fig. 3). The estimated coefficients for the functions are close to the ones of the actual network, another indicator of the goodness of fit. If we increase $\delta$ and $w$ or decrease $c$, the simulated network can produce more matched edges, but also generate more unmatched edges. Therefore, the simulated network in Fig. 7 is considered having the best fit with the actual network based on the $F_{1}$ score among all the trial-and-error tests conducted. 
Table 1. Spearman rank-order correlation coefficients given different hypotheses.

\begin{tabular}{lcc}
\hline \hline Hypothesis & Spearman coefficient & Significance level \\
\hline Our model & 0.53 & 0.01 \\
\hline $\begin{array}{l}\text { Random-link hypothesis: } \\
\text { Random seeds (average over 100 times) }\end{array}$ & 0.02 & \\
\hline
\end{tabular}

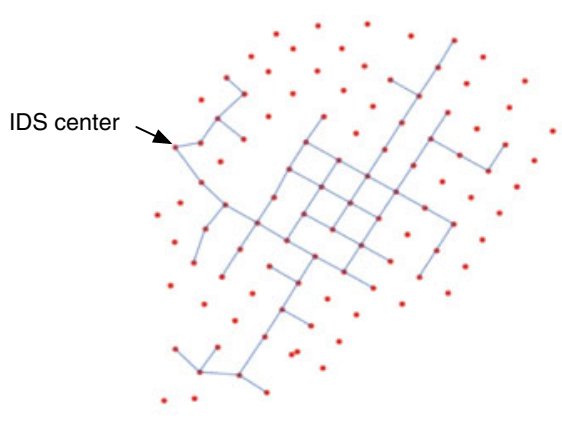

(a)

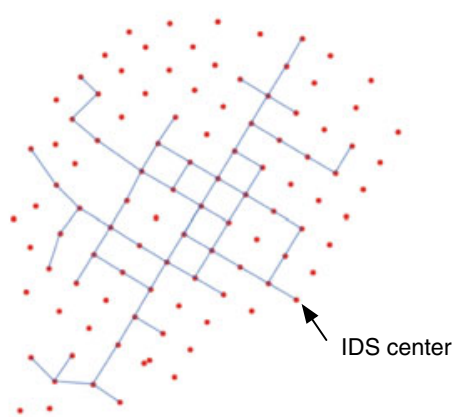

(b)

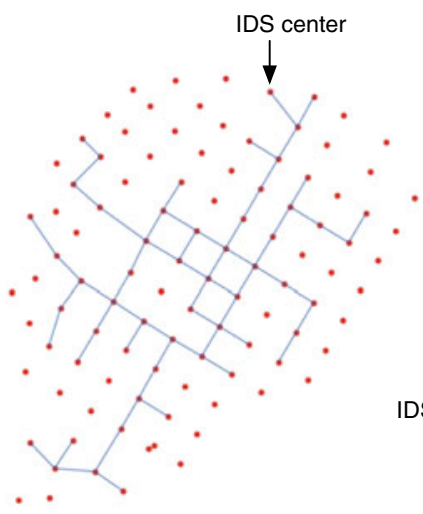

(c)

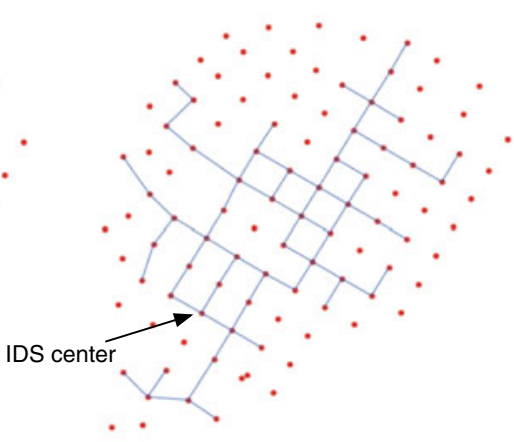

(d)

Fig. 9. The skyway network after we exchange the office size of the IDS Center with the buildings on the periphery of the network. (a) Network after exchanging the IDS Center with a building in the west of downtown. (b) Network after exchanging the IDS Center with a building in the east of downtown. (c) Network after exchanging the IDS Center with a building in the north of downtown. (d) Network after exchanging the IDS Center with a building in the south of downtown.

We also compare the rank order in reality and the rank order in simulation. In Fig. 8, we can observe a positive correlation between the two. Using the Spearman rank-order correlation test [45], we find that the Spearman rank-order correlation between the sequence of the simulated results and the actual sequence equals 0.53 and is statistically significant at the 1 percent significance level. To examine the robustness of the correlation test, we test an alternative hypothesis where a skyway segment is randomly generated, given the same number of edges in the network. We repeat the network growth process 100 times with different random seeds, and calculate the average Spearman rank-order coefficient. As shown in Table 1, the average Spearman coefficient for 100 times of random selection tests equals 0.02 and is not statistically 
significant. In contrast, the simulated rank order shows consistency with the actual rank order in observation.

Furthermore, it is of interest to see how the location of the IDS Center impacts the network topology. We exchange the floor area of the IDS Center with buildings on the eastern, southern, western, and northern periphery of downtown and run the experiments. Figure 9a shows that when the "IDS Center" sits on the western periphery, its adjacent buildings become more connected and there are fewer edges in other zones. When we move the IDS Center's floor area to the eastern periphery, some new edges emerge to connect this "IDS Center", with the loss of edges in the center of downtown (Fig. 9b). A similar trend can be identified in scenarios where the "IDS Center" is located in the north (Fig. 9c) or south (Fig. 9d). The results posit that the locations of important vertices influence the network structure and how the network grows.

\section{Conclusions}

This paper empirically reviews the network structure and the growth of the downtown Minneapolis skyway network. The network structure evolved from tree-like to grid-like and the mean centralities of the vertices decreased over time. Further, the distribution of existing skyway segments is found to follow a power-law function of the mean degree, closeness, and eigenvector centralities of the vertices. The network was built by individual business owners through decentralized decision-making. The value of building a segment was associated with better accessibility for pedestrians, meaning higher rents for businesses on the second floor. We find that buildings with greater accessibility were more likely to be connected earlier: smaller office buildings tended to be connected later when the value of accessibility from the whole network increased. We hypothesize that the network building process is a decentralized decision-making process in both temporal and spatial terms. It has positive and negative feedbacks, the interactions of which generate proper conditions for edges being built.

To illustrate the framework, we propose an agent-based model to replicate this evolutionary process using gravity-based accessibility as the incentive for network growth. For an individual owner, whether a skyway segment should be built depends upon whether the incentive is greater than the disincentive. Our simulation results reveal that this model reproduces well the actual network structure and the evolution of network topology. Overall, the conceptual framework and the exemplary model suggest that (1) understanding the context and history of a transportation system provides unique insights into the network modeling process; (2) the evolution of a transportation system is closely connected with land use which can incentivize or discourage network growth; (3) the locations of important vertices can affect network structure and its evolutionary pattern.

Future work of this research includes incorporating anecdotal or policy factors that may have affected the sequence of the skyway segments being built and investigating whether there existed strategic partnerships among building owners in the midst of competition. It is also of interest to evaluate the relationship between the buildings' rental prices and the network structure.

\section{References}

1. R. Guimerà, L. Amaral, Eur. Phys. J. B 36, 381 (2004)

2. R. Guimerà, S. Mossa, A. Turtschi, L. Amaral, Proc. Natl. Acad. Sci. 102, 7794 (2005)

3. K. Seaton, L. Hackett, Phys. A 339, 635 (2004)

4. P. Sen, S. Dasgupta, A. Chatterjee, P. Sreeram, G. Mukherjee, S. Manna, Phys. Rev. E 67, $36106(2003)$ 
5. V. Latora, M. Marchiori, Phys. A 314, 109 (2002)

6. A. Schadschneider, W. Knospe, L. Santen, L. Schreckenberg, Physica A 346, 165 (2005)

7. A. Masucci, D. Smith, A. Crooks, M. Batty, Eur. Phys. J. B 71, 259 (2009)

8. S. Lämmer, B. Gehlsen, D. Helbing, Physica A 363, 89 (2006)

9. D. Levinson, B. Yerra, Trans. Sci. 40, 179 (2006)

10. M. Corbett, F. Xie, D. Levinson, Environ. Planning B 36, 711 (2009)

11. F. Xie, D. Levinson, Evolving Trans. Networks (Springer, 2011), p. 294

12. S. Dorogovtsev, J. Mendes, Adv. Phys. 51, 1079 (2002)

13. D.J. Price, Science 149, 510 (1965)

14. A. Barabási, R. Albert, Science 286, 509 (1999)

15. O. Frank, D. Strauss, J. Amer. Stat. Asso. 81, 832 (1986)

16. S. Wasserman, P. Pattison, Psychometrika 61, 401 (1996)

17. M. Gastner, M. Newman, J. Stat. Mech. 1, 01015 (2006)

18. F. Schweitzer, W. Ebeling, H. Rose, O. Weiss, Evolutionary Comput. 5, 419 (1997)

19. D. Yamins, S. Rasmussen, D. Fogel, Networks Spat. Econ. 3, 69 (2003)

20. M. Gastner, M. Newman, Eur. Phys. J. B 49, 247 (2006)

21. M. Los, C. Lardinois, Trans. Res. Pt. B 16, 89 (1982)

22. P. Steenbrink, Optimization of transport networks (John Wiley \& Sons, 1974), p. 342

23. M. Barthélémy, A. Flammini, Phys. Rev. Lett. 100, 138702 (2008)

24. D. Helbing, F. Schweitzer, J. Keltsch, P. Molnár, Phys. Rev. E 56, 2527 (1997)

25. D. Helbing, J. Keltsch, P. Molnár, Nature 388, 47 (1998)

26. B. Yerra, D. Levinson, Ann. Reg. Sci. 39, 541 (2005)

27. M. Batty, Y. Xie, Z. Sun, Comput. Environm. Urban Sys. 23, 205 (1999)

28. M. Barthélémy, Phys. Rev. E 68, 026104 (2003)

29. A. Barrat, M. Barthélémy, A. Vespignani, J. Stat. Mech., P05003 (2005)

30. S. Boccaletti, V. Latora, Y. Moreno, M. Chavez, D.-U. Hwang, Phys. Rep. 424, 175 (2006)

31. M. Barthélémy, Phys. Rep. 499, 1 (2011)

32. F. Xie, D. Levinson, Networks Spat. Econ. 9, 291 (2009)

33. D. Levinson, J. Trans. Land Use 4, 1 (2011)

34. J. Fox, R. Rindfuss, S. Walsh, V. Mishra, People and the Environment (Kluwer, 2003), p. 344

35. S. Manson, T. Evans, Proc. Natl. Acad. Sci. 104, 20678 (2007)

36. J.P. Byers, Breaking the Ground Plane, Ph.D. thesis, Univ. of MN, 1998

37. M. Kramer, Minnesota Leasing Guide (Law Bulletin, Chicago, IL, 2004)

38. J. Buhl, J. Gautrais, R.V. Solé, P. Kuntz, S. Valverde, J. Deneubourg, G. Theraulaz, Eur. Phys. J. B 42, 123 (2004)

39. S. Wasserman, K. Faust, Social Network Analysis: Methods and Applications (Cambridge University Press, 1994)

40. G. Sabidussi, Psychometrika 31, 581 (1966)

41. L.C. Freeman, Social Networks 1, 215 (1979)

42. P. Bonacich, Amer. J. Sociol. 92, 1170 (1987)

43. A. El-Geneidy, L. Kastelberger, H. Abdelhamid, J. Trans. Land Use 4, 33 (2011)

44. C.J. van Rijsbergen, Information Retrieval (Butterworth-Heinemann, 1979)

45. C. Spearman, Amer. J. Psychol. 15, 72101 (1904) 